\title{
Yod
}

Revue des études hébraïques et juives

$16 \mid 2011$

Le yiddish dans la sphère francophone

\section{Langue juive ou jargon : Les dénominations du yiddish en Pologne avant 1939}

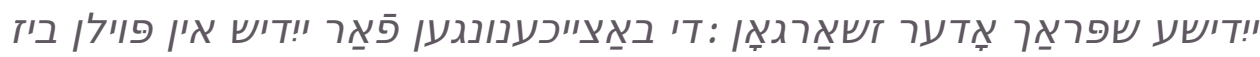

\section{Natalia Krynicka}

\section{(2) OpenEdition}

1 Journals

\section{Édition électronique}

URL : https://journals.openedition.org/yod/281

DOI : $10.4000 /$ yod. 281

ISSN : 2261-0200

Éditeur

INALCO

\section{Édition imprimée}

Date de publication : 1 juin 2011

Pagination : 99-117

ISBN : 978-2-85831-191-0

ISSN : 0338-9316

\section{Référence électronique}

Natalia Krynicka, «Langue juive ou jargon : Les dénominations du yiddish en Pologne avant 1939 », Yod [En ligne], 16 | 2011, mis en ligne le 06 décembre 2011, consulté le 08 juillet 2021. URL : http:// journals.openedition.org/yod/281 ; DOI : https://doi.org/10.4000/yod.281

Ce document a été généré automatiquement le 8 juillet 2021.

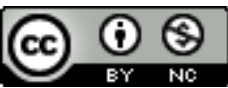

Yod est mis à disposition selon les termes de la Licence Creative Commons Attribution - Pas

d'Utilisation Commerciale 4.0 International. 


\title{
Langue juive ou jargon : Les dénominations du yiddish en Pologne avant 1939
}

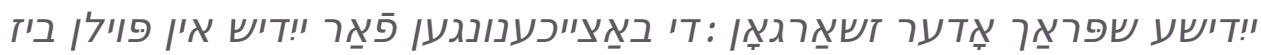

\author{
Natalia Krynicka
}

1 Toute la complexité de l'attitude envers le yiddish des yiddishophones et des polonophones, des Juifs ou des non-Juifs, dans la Pologne d'avant la deuxième guerre mondiale se reflète dans la dénomination de la langue. Celle-ci évolue au fil des années, en suivant souvent la terminologie internationale, notamment allemande. Loin d'être une simple question linguistique, elle dévoile de profonds clivages identitaires et concerne des choix idéologiques et politiques.

\section{La dénomination neutre}

2 L'appellation la plus ancienne mais aussi la plus neutre est «jæ̨zyk żydowski » ou «żydowski » tout court, ce qui signifie « langue juive ». Ceci correspond exactement à l'appellation moderne du yiddish par ses locuteurs, le mot «yidish» étant un adjectif dérivé du substantif « yid » - « Juif ».

D'après l'éminent linguiste Max Weinreich, la dénomination «yidish» au sein du yiddish même apparaît par écrit au milieu du XVII ${ }^{e}$ siècle et remplace progressivement les appellations antérieures: «loshn-ashkenaz ( «langue ashkénaze »), «taytsh» (" allemand») ou "yidish-taytsh» (" judéo-allemand»). En Autriche, l'équivalent " jüdische Sprache » (" langue juive ») est courant à partir de la fin du XVIII ${ }^{\mathrm{e}}$ siècle, et à Prague, en tchèque, «židovske » s'emploie dès la fin du XVI e siècle. En Angleterre et plus tard aux États-Unis, les immigrés juifs apportent le nom "yidish» à partir du milieu du XIX ${ }^{e}$ siècle. Il sera transcrit en caractères latins de la manière répandue aujourd'hui dans plusieurs pays, "yiddish ». Dans l'aire germanophone, le même nom se répand à partir de la première guerre mondiale avec l'orthographe «jiddisch $»^{1}$. 
Auparavant, les appellations les plus répandues étaient «jüdisch-deutsch» et "Judendeutsch».

\section{Les dénominations péjoratives}

$\mathrm{Au}$ cours du XIX ${ }^{\mathrm{e}}$ siècle et au moins jusqu'à l'indépendance de la Pologne en 1918, la dénomination «język żydowski » est concurrencée par celle de "żargon » (jargon) ou «żargon żydowski » (jargon juif), empruntée à l'allemand ${ }^{2}$. Ce sont les adeptes de la Haskala berlinoise (Lumières juives), partisans de l'apprentissage de la langue du pays, en l'occurrence de l'allemand, qui ont adopté ce mot d'origine française à la fin du $\mathrm{XVIII}{ }^{\mathrm{e}}$ siècle comme une désignation condescendante du yiddish. Il sera ensuite repris dans divers autres pays européens.

Bien que péjorative à l'origine, car refusant d'emblée au yiddish le statut de langue, cette appellation devient relativement neutre en Europe de l'Est. Jusqu'au début du XX siècle, elle est même utilisée en yiddish par des journalistes et écrivains, comme Yankev Dinezon (1856-1919) ou Sholem-Aleykhem (1859-1916), l'un des pères de la littérature yiddish moderne. Ce mot à consonance étrangère n'est pas toujours perçu comme condescendant, et a l'avantage d'éviter d'éventuels malentendus causés par le double sens du nom « yidish » dans cette langue - «juif » et « yiddish » à la fois. Dans l'aire russophone, cette ambiguïté est probablement doublée par celle de l'emploi du mot russe "yevreyskiy », qui peut signifier "juif», "yiddish» et même « hébreu ». Tandis que dans la deuxième moitié du XIX ${ }^{\mathrm{e}}$ siècle, le mot "yevreyskiy » est réservé à l'hébreu, et «novo-yevreyskiy » (avec l'adjectif «neuf») ou " razgovorno-yevreyskiy » (avec l'adjectif "parlé ») désigne le yiddish, au fil des années apparait une tendance à appeler l'hébreu "drevne-yevreyskiy " (avec l'adjectif "ancien») et le yiddish " yevreyskiy » tout court, jusqu'à ce que l'on commence à employer le terme «idish » après la révolution de $1917^{3}$

6 Une autre raison d'utiliser la désignation «zhargon » au sein même des milieux yiddishophones, est l'attitude traditionnelle envers cette langue. Depuis ses origines, elle est considérée comme une langue vernaculaire subordonnée à l'hébreu, la langue sacrée, qui, elle, mérite toute l'attention ${ }^{4}$. Le refus catégorique du yiddish de la part de la Haskala berlinoise affaiblit encore son statut parmi l'intelligentsia juive du XIX ${ }^{\mathrm{e}}$ siècle. Au départ, les auteurs d'inspiration maskilique en Europe centrale et orientale n'utilisent le yiddish que pour des raisons pragmatiques, afin de diffuser leurs idées au sein des larges masses juives. Ce n'est que très progressivement que le yiddish devient une valeur en soi. Il faut attendre le début du $\mathrm{XX}^{\mathrm{e}}$ siècle et la cristallisation du mouvement yiddishiste pour que le mot « zhargon » reprenne son caractère péjoratif à l'oreille des yiddishophones et qu'ils passent définitivement à la désignation " yidish ».

7 En polonais, la dénomination "żargon" pour désigner le yiddish, ne semble se généraliser que dans le dernier quart du XIX ${ }^{e}$ siècle. L'entrée « żargon » ne figure pas dans le dictionnaire de Linde (1812, $2^{\mathrm{e}}$ édition 1861$)^{5}$; dans l'encyclopédie d'Orgelbrand de 1868 , on le retrouve dans un emploi équivalent au français de l'époque, sans référence aucune au yiddish ${ }^{6}$. En revanche, lorsque parait la Grande Encyclopédie illustrée (1890), le terme « żargon » est bien ancré dans la langue pour désigner le yiddish. Dans l'article consacré à l'écrivain yiddish Mendele Moykher-Sforim (pseud. de SholemYankev Abramovitsh, 1836-1917) dont deux romans ont été traduits en polonais dans les années 1885-1886, les termes "autor żargonowy" et "literatura żargonowa » 
(" auteur écrivant en jargon » et « littérature en jargon ») ne semblent pas comporter de nuance péjorative ${ }^{7}$.

8 Ce n'est pas le cas de l'Encyclopédie de la Mère Patrie polonaise publiée à Lemberg en 1905-1907, où les références aux Juifs sont souvent accompagnées de remarques antisémites. Le terme "żargon 》 y est employé avec une connotation péjorative sans aucune ambiguïté :

'Jargon' signifie langue pervertie. La majorité des Juifs polonais, bien qu'établie sur notre terre depuis des centaines d'années, parle une langue allemande pervertie, ou bien le polonais perverti-donc le jargon ${ }^{8}$.

Cette définition impitoyable non seulement condamne tous les Juifs polonais pour leur manque d'assimilation linguistique, sous-entendu de patriotisme, mais refuse au yiddish jusqu'à son identité propre, en le rangeant dans la catégorie des erreurs linguistiques à côté du polonais déformé, et le ramène ainsi au sens primaire du mot «jargon ».

10 Utilisée par les antisémites, la désignation «żargon» est donc progressivement abandonnée dans le milieu juif d'expression polonaise. Toutefois, il arrive que des assimilationnistes, même ceux de la deuxième génération, continuent à employer le mot jargon, avec tout le bagage d'hostilité hérité de leurs pères. L'abandon progressif de cette désignation dans ces milieux est suivi - quoique beaucoup plus lentement et non sans réticences - par le milieu non juif.

11 Ainsi, en 1915, le philologue Jakub Willer, auteur de l'article sur le yiddish dans l'Encyclopédie polonaise, se voit imposer contre son gré par l'éditeur le titre «Le jargon juif dans les territoires polonais ", alors qu'il penche plutôt pour "Le dialecte juif » ("gwara żydowska ») ${ }^{10}$. Dès le début de l'article, il précise d'ailleurs que l'appellation « żargon » est condescendante ${ }^{11}$.

12 À partir du retour à l'indépendance de la Pologne en 1918, le terme « język żydowski » se répand à nouveau pour désigner le yiddish, et ceux qui continuent à employer la dénomination «żargon » le font avec une nuance nettement péjorative. C'est le cas du dictionnaire de Michał Arct de 1929, où la définition du mot «żargon » rappelle en plusieurs points celle de l'Encyclopédie de la Mère Patrie polonaise, publiée plus de vingt ans plus tôt :

Jargon (du français) : langage corrompu par des mots déformés, utilisé dans les villes;

dialecte allemand corrompu, utilisé par la populace juive de Pologne ${ }^{12}$.

13 Le fait de répéter les anciennes formulations (" allemand corrompu») et l'association du yiddish à la " populace juive ", terme péjoratif en soi qui suggère que le yiddish ne peut pas être une langue de culture, témoignent des préjugés antisémites de l'auteur.

14 Une partie de la classe politique polonaise continue également à utiliser délibérement le mot «żargon». En 1925, dans une lettre à Nasz Przegląd, concernant la langue d'enseignement dans les établissements pour enfants juifs, le ministre d'éducation Stanisław Grabski expose ses opinions sur le yiddish :

Personnellement, je crois que le jargon est un épisode historique, tandis que la langue hébraïque a un passé et un avenir dans l'histoire de la culture juive. Par conséquant, de la part de l'éducation nationale, l'hébreu mérite plus de considération que la soi-disant langue juive [żydowski] ${ }^{13}$. appeler le yiddish par le nom de «żydowski ». Selon lui, l'appellation «langue juive » 
conviendrait à l'hébreu, «véritable » langue juive, plutôt qu'au yiddish, qui ne mérite pas mieux que le nom de « jargon ».

Au-delà de ces utilisations délibérément dépréciatives voire péjoratives, l'emploi du mot « żargon » et encore plus de l'adjectif « żargonowy » résulte souvent de l'ignorance ou de la maladresse. Ainsi l'Encyclopédie illustrée publiée au cours des années 1920 contient l'entrée «żargon $»^{14}$ sans référence au yiddish, tandis que dans l'entrée "littérature hébraïque ${ }^{15}$, on emploie bien ce mot pour désigner le yiddish. Dans les années 1930, l'Encyclopédie du XXe siècle cite d'Abramovitsh (Mendele Moykher-Sforim) comme un "romancier hébraïco-jargonique ( " powieściopisarz hebrajskożargonowy »1 ${ }^{16}$. On peut imaginer que dans ce dernier cas, l'auteur de l'article n'a pas trouvé d'autre adjectif et que le terme «żydowski » (« yiddish » mais aussi «juif») lui semblait trop ambigu.

17 À côté du terme "żargon żydowski», on retrouve également celui de «żargon żydowsko-niemiecki » («jargon judéo-allemand $)^{17}$ ou « żargon niemiecki » (« jargon allemand ») ${ }^{18}$, l'équivalent du «jüdisch-deutsch» (anciennement «jüdisch-teutsch») utilisé communément en allemand à partir $\mathrm{du} \mathrm{XVI}{ }^{\mathrm{e}}$ siècle. En polonais, cette appellation a un double sens : elle peut juste viser la précision pour éviter une éventuelle confusion avec l'hébreu, mais elle souligne avant tout le caractère "étranger» du parler juif polonais face à la langue majoritaire du pays. Dans la Pologne issue des partages (1772-1918) et peu après, cette dénomination est donc doublement péjorative, car elle associe les Juifs aux occupants allemands et autrichiens, et le yiddish à l'allemand, langue imposée aux Polonais, souvent avec violence, notamment dans les territoires annexés par la Prusse. À une époque où les nationalismes se cristallisent et l'indépendance du pays est un sujet central pour la majorité des intellectuels, la question linguistique devient un choix politique. Alors même qu'on chante le poème de Maria Konopnicka «L'Allemand ne nous crachera pas au visage, il ne germanisera pas nos enfants ${ }^{19}$, l'association directe du yiddish à l'allemand équivaut à accuser ses locuteurs d'être des traîtres à la nation.

Des variantes de cette appellation, «zepsuta niemczyzna » («allemand perverti ») ou "pokaleczona niemczyzna » ("allemand écorché »), sont également très répandues. Elles aussi provienent du terme allemand ("verdorbenes Deutsch»). Dans cette désignation, les associations évoquées plus haut sont encore renforcées - on ne parle plus d'une sorte d'allemand, mais d'allemand tout court. En outre, l'incorrection devient un trait essentiel du parler des Juifs ashkénazes. On le voit par exemple dans la façon dont les héros « positifs » du premier roman polonais consacré entièrement à la thématique juive, Lejbe et Siora de Julian Ursyn Niemcewicz (1821) parlent du yiddish :

Et que pourrais-je exprimer dans cet allemand écorché, noté en caractères hébraïques, que nous appelons langue juive? Ni Abraham, ni Isaac, ni Jacob ni notre législateur Moïse n'ont jamais parlé cette langue. La langue maternelle de tout un chacun est celle de la terre où il est né: nous sommes nés en Pologne, par conséquent c'est le polonais qui est notre langue maternelle. C'est dans cette langue que nous devons écrire et parler ${ }^{20}$.

19 Comme on le voit, ici le terme a été utilisé pour démontrer le caractère étranger du yiddish, non seulement par rapport à la Pologne, mais aussi par rapport aux Juifs euxmêmes et à leur histoire.

Un autre terme clairement péjoratif désignant un langage jugé inintelligible et désagréable à l'oreille a connu une évolution similaire à celle de «żargon ». Il s'agit du substantif «szwargot» («baragouin») et du verbe qui en est dérivé "szwargotać », 
utilisés parfois pour parler du yiddish dans la prose $^{21}$ ou même dans la poésie ${ }^{22}$ polonaise, et surtout, bien sûr, dans la littérature antisémite ${ }^{23}$. On le retrouve également dans le journal juif assimilationniste Izraelita (1866-1912) dans les années $1880^{24}$, ce qui confirme l'attitude pour le moins condescendante de ce périodique envers le yiddish. Par la suite, cette dénomination disparaît d'Izraelita avec le départ de son rédacteur en chef Samuel Cwi (Henryk) Peltyn, mort en $1896^{25}$.

Dans le dictionnaire polonais de Linde (1812), le terme "szwargot» désigne tout langage inconnu, désagréable ou incompréhensible, notamment les différentes langues non slaves que l'on pouvait entendre en Pologne : le latin, l'allemand et le français ${ }^{26}$. On n'y trouve aucune référence au yiddish, et le verbe "szwargotać » n'est associé à aucune langue en particulier. Cent ans plus tard, le dictionnaire de Karłowicz, Kryński et Niedźwiedzki (1912) reprend la définition et les exemples de Linde, mais y ajoute également les Juifs, sans toutefois préciser de quelle langue il s'agit: «Les Juifs, les Souabes [ici : les Allemands], les marchandes baragouinent (« szwargoczĄ ») ${ }^{27}$. Dans le même dictionnaire, les adjectifs "szwargotliwy » et "szwargotny " sont également associés aux Juifs, et le terme "szwargot» est un élément de définition du terme «żargon $»^{28}$. Désormais le mot « szwargot » semble donc se référer surtout aux langues germaniques, et particulièrement au yiddish.

Dans le dictionnaire d'Arct (1929), dans la définition de "szwargot», les exemples d'autres langues ont disparu, seul le yiddish est resté, devenu quasiment le synonyme de «szwargot»: «Baragouin: langage guttural, désagréable et inintelligible, spécialement le jargon juif $»^{29}$. Le même terme est utilisé par Arct dans l'entré «żydowski » pour désigner le yiddish: «langue juive - l'hébreu ou le baragouin (szwargot) des Juifs polonais $»^{30}$.

23 Cette désignation stigmatise donc d'emblée le yiddish comme inesthétique, assimilé à une nuisance sonore, mais également à un langage insensé et incompréhensible par excellence. Dans le dictionnaire d'Arct, qui comporte, comme on l'a déjà vu, des traits antisémites, cette impression est volontairement renforcée par l'emploi du terme « jargon » pour le yiddish.

\section{La prise de conscience nationale et linguistique}

À partir de la fin du XIXe siècle, la conscience nationale juive se développe à une vitesse croissante. Ceci va de pair avec une réflexion sur la langue nationale juive. Les " hébraïstes " s'affrontent avec les «yiddishistes » à propos du choix de la langue. En 1908, la conférence linguistique de Czernowitz (aujourd'hui Tshernivtsi en Ukraine), conçue au départ pour discuter de différents aspects de la grammaire et de la stylistique du yiddish, se transforme en arène où débatent passionnément les partisans du yiddish et ceux de l'hébreu. Finalement, le yiddish est proclamé non pas « la », mais "une» langue nationale juive. Cette déclaration est quand même d'une importance capitale pour le futur épanouissement du yiddish. En premier lieu, après des siècles de dénominations condescendantes, il est enfin reconnu comme une langue à part entière. Ensuite, le statut d'une langue nationale juive, même avec l'article indéfini, lui procure un nouveau rang au sein de la société juive, et aussi dans ses contacts avec les milieux non juifs. 

opposés à l'assimilation et assumant une identité nationale juive, se sentent concernés par la terminologie utilisée pour désigner le yiddish et, plus largement, par l'attitude des autorités et de l'opinion publique en Pologne envers cette langue. C'est particulièrement le cas du quotidien varsovien Nasz Przegląd (1923-1939), le plus important périodique juif de langue polonaise en Pologne de l'entre-deux-guerres ${ }^{31}$. Bien que d'orientation plutôt sioniste (c'est le mouvement pour lequel il préconise de voter pendant les élections communautaires, municipales et parlementaires), le journal est très engagé pour le respect des droits du yiddish. Il proteste à chaque fois que surgissent des cas de discrimination officielle de cette langue, héritée de l'époque de la Pologne partagée ${ }^{32}$ et milite - en vain - pour la faire bénéficier de tous les droits d'une langue minoritaire, à côté de l'ukrainien et du biélorusse, sur les territoires des confins orientaux $^{33}$. Il est également bienveillant vis-à-vis des efforts ponctuels des représentants juifs (notamment du Bund et Poalei-Tsion) pour introduire le yiddish dans les débats des conseils municipaux ${ }^{34}$. Quand un bureau de télégraphe à Varsovie refuse d'accepter des telégrammes en yiddish (en caractères latins) arguant qu'à part les langues européennes, seul le japonais et l'hébreu y sont autorisées, Nasz Przegląd s'insurge et remarque que le yiddish fait justement partie des langues européennes ${ }^{35}$. Le journal réagit aussi fermement quand des instances culturelles et politiques utilisent des désignations péjoratives du yiddish. À la sortie de deux encyclopédies polonaises, Mark Turkov, écrivain bilingue yiddish-polonais, déplore dans Nasz Przegląd l'usage du mot «żargon» pour désigner le yiddish, tout en employant lui-même le terme «żydowski $»^{36}$. Le rédacteur en chef du journal, Jakub Appenszlak, s'insurge contre une émission de radio en polonais, où il a entendu que les Juifs se servaient du jargon, composé de mots russes, polonais et en partie hébreux, tandis que l'hébreu était une langue de science et littérature :

[...] donc tout va bien. La Radio polonaise, qui raconte à la Pologne et au monde des histoires antisémites à dormir debout, n'a jamais entendu parler du yiddish [żydowski], la langue d'Asch, de Sholem-Aleykhem, Peretz, Leyvik. Pour la radio polonaise, il n'y a pas de langue juive d'une riche culture, d'un enseignement diversifié, de la science et du théâtre. Le monde de culture et de politique a presque unanimement reconnu cette langue et ses droits - il n'y a que sur les ondes de Varsovie que cela reste 'un jargon polono-russo-hébreu'37.

Sporadiquement, on entend des voix de protestation contre cette appellation également de la part des non-Juifs. Ceci dit, leurs prises de position sont publiées elles aussi dans les journaux juifs en langue polonaise, et non pas dans la presse générale. C'est au journaliste de Nasz Przegląd que T. Hołówka, le directeur de l'Institut des recherches sur les minorités nationales en Pologne (Instytut badań spraw mniejszości narodowych w Polsce), confie :

La société polonaise se contente de généralités, très souvent des époques passées. En ce qui concerne p. ex. la minorité juive, la société polonaise traite avec dédain la langue yiddish, connue parmi les Polonais sous la désignation condescendante de 'jargon'38. 

moderne »), l'équivalent de "novoyevreyskiy » en russe. Cette appellation est neutre et son rôle est avant tout d'éviter la confusion avec le mot ambigu "żydowski » ${ }^{39}$. Dans la deuxième moitié des années 1920, dans les publications en langue polonaise on voit apparaître sporadiquement le terme " yiddish ». Il est orthographié « idysz », « jidysz » ou, comme en anglais, "yiddish » et décliné selon les règles de la grammaire polonaise, p. ex. « $z$ idyszu » ou même « $z$ Yiddish'u $»^{40}$. On note également la présence de l'adjectif «jidyszowy ». On l'emploie soit pour éviter la confusion entre le nom de la langue et l'adjectif « juif » ${ }^{41}$, soit comme une marque de respect, de conscience linguistique. Il est utilisé surtout par les Juifs yiddishistes et anti-assimilationnistes, plus rarement par les sionistes. On peut imaginer que certains sionistes emploient le terme "yiddish » en polonais précisément pour éviter l'appellation « langue juive ».

Au fil des années, on observe une lente progression de cette appellation, y compris dans les milieux non juifs. Le mot «idysz " apparaît par exemple dans l'encyclopédie Le Monde et la vie éditée à partir de 1933 sous la forme de cahiers mensuels. En 1934, on le voit dans l'article « langue » de cette encyclopédie, au chapitre « langue nationale face au dialecte » écrit par Kazimierz Nitsch, professeur de linguistique à l'Université Jagiellone de Cracovie et spécialiste en dialectologie :

La langue des Juifs polonais est en principe, de par son vocabulaire et sa grammaire, un dialecte du haut-allemand. Cependant, étant le moyen d'expression d'une population culturellement très distincte, dont un des aspects est la multitude de mots hébraïques, sans parler du nombre de mots slaves, et de l'alphabet hébraïque distinct, il peut être tout à fait considéré comme une langue. Celle-ci est de plus en plus souvent appelée non pas 'jargon' (ce qui signifie uniquement 'mélange') mais, pour ne pas la confondre avec l'hébreu : langue juive, jidisz, etc ${ }^{42}$.

En 1939, le terme «idysz » apparaît pour la première fois en tant qu'entrée séparée dans le même ouvrage encyclopédique. Les dénominations «żargon» et «żydowski język » y apparaissent également, mais renvoient toutes les deux à « idysz » :

Idysz (appelé couramment jargon juif) - la langue de la majorité des Juifs d'Europe de l'Est et des milieux de leur émigration (Londres, Paris, États-Unis, Argentine). Il est apparu suite à l'union de quelques dialectes du vieil allemand avec des éléments hébraiques, slaves et romans. Ses débuts remontent au $X^{e}$ siècle. Le idysz utilise l'alphabet hébraïque. Depuis quelques décénnies une abondante littérature s'est développée dans cette langue ${ }^{43}$.

31 Cette définition, bien différente de celle du dictionnaire d'Arct publié dix ans auparavant, marque une véritable rupture avec l'ancienne approche du yiddish. Celuici commence enfin à être considéré impartialement, comme n'importe quelle autre langue. C'est la preuve qu'à la veille de la deuxième guerre mondiale, malgré l'antisémitisme ambiant, les mentalités changent petit à petit du côté polonais en ce qui concerne le yiddish. Ceci correspond à une évolution similaire dans plusieurs pays européens (notamment en Union soviétique, où dès le début le yiddish est considéré comme une langue à part entière et constitue l'objet de recherches poussées), ainsi qu'aux États-Unis.

On observe donc, rien qu'en consultant les dictionnaires des $\mathrm{XIX}^{\mathrm{e}}$ et $\mathrm{XX}^{\mathrm{e}}$ siècles, une polarisation progressive de la terminologie concernant le yiddish. À côté des désignations neutres, se développent dans la deuxième moitié du XIX ${ }^{e}$ siècle des termes nettement péjoratifs. On remarque d'ailleurs que le clivage entre les termes neutres et dépréciatifs ne correspond pas exactement à celui qui sépare auteurs juifs et non juifs, mais que la réalité est beaucoup plus complexe. Le processus de radicalisation 
s'accélère vers la fin du XIX et le début du XX ${ }^{e}$ siècle. À cette époque, la dénomination péjorative est abandonnée progressivement par les milieux juifs polonophones, et elle est de plus en plus associée aux idées antisémites. Dans l'entre-deux-guerres, la polarisation atteint son apogée : tandis que les milieux antisémites persévèrent souvent dans leurs appellations condescendantes, les milieux yiddishistes et une partie des Juifs polonophones choisissent une terminologie neutre voire valorisante. Vers la fin des années 1930, celle-ci commence à s'étendre dans les milieux non juifs.

\section{BIBLIOGRAPHIE}

BAUMGARTEN, Jean (1993), Le yiddish, Que sais-je ?, PUF, $2^{\mathrm{e}}$ éd.

BAUMGARTEN, Jean, ERTEL, Rachel, NIBORSKI, Itzhok [et al.] (dir.) (1998), Mille ans de cultures ashkénazes, Paris, Liana Levi.

BECHTEL, Delphine (2002), La renaissance culturelle juive en Europe centrale et orientale 1897-1930: langue, littérature et construction nationale, Paris, Belin.

Encyklopedya : zbiór wiadomości z wszystkich gałĘzi wiedzy, wydanie Macierzy Polskiej, wyd. 2, pomnożone i ilustrowane, Lwów, nakł. Wydawnictwa Macierzy Polskiej, 1907

Encyklopedyja powszechna, Warszawa, Nakł., druk i własność S. Orgelbranda, 1868

Encyklopedja Ultima Thule, pod redakcją Dra St. Fr. Michalskiego, Warszawa, 1927

ERTEL, Rachel (2003), Brasier de mots, Paris, Liana Levi.

GUTMAN, Yisrael, MENDELSOHN, Ezra, REINHARZ, Jehuda \& SHMERUK, Chone (éds) (1989), The Jews of Poland between two world wars, Hanover (NH), University Press of New England.

Ilustrowana Encyklopedja Trzaski, Everta i Michalskiego, opracowana pod redakcją Dra Stanisława Lama, nakł. KsiĘgarni Trzaski, Everta i Michalskiego, Warszawa, cop. 1928

KRAKOWSKI, I. (1928), Słownik polsko-żydowski/Poylish-yudish verterbukh, Warszawa, Sprachen.

LIFSIC, M. (1876), Novoevrejsko-russkij slovar, Zitomir.

LIFSIC, M. (1869), Russko-novoevrejskij slovar, Zitomir.

MARCUS, Joseph (1983), Social and Political History of the Jews in Poland: 1919-1939, New York, Mouton.

MAREK, Arn (1929), Pełny słownik polsko-żydowski, zestawiony według najnowszych źródeł, Warszawa, wyd. Achisefer przy Tow. Wyd. Central.

NIBORSKI, Yitskhok, VAISBROT, Bernard (2002), Dictionnaire yiddish-français, Paris, Bibliothèque Medem.

NIGER, Shmuel \& SHATSKI, Yankev (éd.) (1956-1981), Leksikon fun der nayer yidisher literatur, t. 1-8, New York, Alveltlekher Yidisher Kultur-Kongres.

PROKOP-JANIEC, Eugenia (1992), MiĘdzywojenna literatura polsko-żydowska jako zjawisko kulturowe i artystyczne, Kraków, Universitas. 
REYZEN, Zalmen (1928), Leksikon fun der yidisher literatur, prese un filologye, t. 1-4, Vilne, B.

Kletskin.

Russko-yevreyskiy (idish) slovar pod redaktsey M. A. Shapiro, I. G. Spivaka i M. Ya. Shulmana, Moskva, Russkiy Yazik, 1984

SHMERUK, Chone (1985), The Esterke Story in Yiddish and Polish Literature: A Case Study in the Mutual Relations of Two Cultural Traditions, Jerusalem, The Zalmen Shazar Center.

SHMERUK, Chone (1988), Prokim fun der geshikhte fun yidisher literatur, Tel-Aviv : Farlag Y. L. Peretz, Yerusholaim : Yidish-Opteylung fun der Hebreisher Universitet.

Sieben Sprachen Wörterbuch, Deutsch, Polnisch, Russisch, Weissrussisch, Litauisch, Lettisch, Jiddisch, Leipzig, 1918

Słownik ilustrowany jĘzyka polskiego M. Arcta, Wyd. M. Arcta w Warszawie, t. 2, wyd. 3, 1929

Słownik jĘzyka polskiego Jana Karłowicza, Adama Kryńskiego i Władysława Niedźwiedzkiego, zeszyt 31-35, ułożony przez Władysława Niedźwiedzkiego z udziałem Kazimierza Króla, Warszawa, nakł. Prenumeratorów i Kasy im. Mianowskiego w druk. Gazety Handlowej, 1912

Słownik jĘzyka polskiego przez M. Samuela Bogumila Linde, w Warszawie, u autora, 1812, 6 vol.; $2^{\mathrm{e}}$ éd.: Lwów, w drukarni Zakładu Ossolińskich, 1861, 6 vol.

STEFFEN, Katrin (2002), Jüdische Polonität? Studien zur polnischsprachigen jüdischen Presse in der Zweiten Polnischen Republik (1918-1936), Inaugural-Dissertation zur Erlangung des Doktorgrades am Fachbereich Geschichts- und Kulturwissenschaften der Freien Universität Berlin, Berlin.

Świat $i$ życie : zarys encyklopedyczny wspótczesnej wiedzy $i$ kultury, red. naczelny : Dr. Zygmunt Łempicki, red. Anna Chorowiczowa, KsiĄżnica - Atlas, Lwów - Warszawa, 1934

Trzaski, Everta i Michalskiego Encyklopedja XX wieku, opracowana pod redakcjĄ Dra Stanisława Lama, nakł. KsiĘgarni Trzaski, Everta i Michalskiego, Warszawa, [1937?]

WEINREICH, Max (1973), Geshikhte fun der yidisher shprakh, New York, YIVO.

Wielka Encyklopedya Powszechna Ilustrowana, Warszawa, nakład i druk S. Sikorskiego, t. 1: A, 1890

WILLER, Jakub (1915), « Żargon żydowski na ziemiach polskich », Encyklopedya polska, nakł. Akademii UmiejĘtności, Gebethner i Wolff, Kraków, vol. 3, partie 3, pp. 395-424.

WOLFSON, M. (1906), Słownik polsko-russko-żydowski, Warszawa, F. Berlinerblau.

WOLFSON, M. (1919), Słownik polsko-żydowski, Warszawa, nouvelle éd. 1923

\section{NOTES}

1. Voir Max Weinreich, Geshikhte fun der yidisher shprakh, New York, YIVO, 1973, t. 1, pp. 321-323.

2. Cf. Weinreich Geshikhte..., op. cit., p. 327.

3. Preuve que la dénomination "yevreyskiy » en russe pouvait prêter à confusion encore dans la deuxième moitié du XX $\mathrm{XX}^{\mathrm{e}}$ siècle, le titre du dictionnaire russe-yiddish, publié en 1984, rajoute le mot «idish" après "yevreyskiy " pour préciser de quelle langue il s'agit, cf. Russko-yevreyskiy (idish) slovar pod redaktsey M. A. Shapiro, I. G. Spivaka i M. Ya. Shulmana, Moskva, Russkiy Yazik, 1984.

4. Voir Chone Shmeruk, Prokim fun der yidisher literatur-geshikhte, Tel-Aviv: Farlag Y. L. Peretz, Yidish-Opteylung / der Hebreisher Universitet in Yerusholaim, 1988, pp. 11-29. 
5. Słownik jĘzyka polskiego przez M. Samuela Bogumiła Linde, w Warszawie, u autora, 1812, 6 vol.; $2^{e}$ éd.: Lwów, w drukarni Zakładu Ossolińskich, 1861, 6 vol.

6. Encyklopedyja powszechna, Warszawa, Nakł., druk i własność S. Orgelbranda, 1868, t. 28, p. 913.

7. «Abramowicz», Wielka Encyklopedya Powszechna Ilustrowana, Warszawa, nakład i druk S. Sikorskiego, t. 1: A, 1890, pp. 64-65.

8. «Żargon znaczy tyle co jĘzyk zepsuty. Żydzi polscy pomimo, że setki lat siedzĄ na ziemi naszej, mówiĄ po wiદ̨kszej czĘści albo zepsutym niemieckim, albo zepsutym polskim jĘzykiem, a wiec żargonem ». Encyklopedya: zbiór wiadomości z wszystkich gałĘzi wiedzy, wydanie Macierzy Polskiej, wyd. 2, pomnożone i ilustrowane, Lwów, nakł. Wydawnictwa Macierzy Polskiej, vol. 2 (M-Z), 1907, p. 1104.

9. Jakub Willer, «Żargon żydowski na ziemiach polskich », Encyklopedya polska, nakł. Akademii Umiejદ̨tności, Gebethner i Wolff, Kraków, [1915], vol. 3, partie 3, pp. 395-424.

10. À propos du caractère imposé du titre, voir Weinreich, Geshikhte..., op. cit., vol. 3, p. 340.

11. Willer, «Żargon żydowski... » op. cit., p. 396.

12. «Żargon fr., zepsuta mowa o wyrazach poprzekrĘcanych, używana w miastach; zepsuty dialekt niemiecki, używany przez pospólstwo żydowskie w Polsce », M. Arcta Słownik ilustrowany jĘzyka polskiego, Wyd. M. Arcta w Warszawie, t. 2, wyd. 3, 1929, p. 1200. Dans ce dictionnaire, voir aussi la définition du mot «żargonowiec» (jargoniste, yiddishiste): "Juif polonais qui parle uniquement en jargon, soit parce qu'il il ne maitrise pas d'autre langue, soit pour des raisons idéologiques, nationaliste juif » («Żargonowiec, Żyd polski, mówiący tylko żargonem, bądź dlatego, że innego nie posiada, bĄdź ze wzglĘdów ideowych, nacjonalista żydowski »), ibid.

13. «Osobiście uważam, że żargon jest epizodem historycznym, gdy j̨̨zyk hebrajski ma przeszłość i przyszłość w historji kultury żydowskiej. Ze strony wiĘc państwowych władz szkolnych zasługuje wiદ̨cej na uwzglĘdnienie jદ̨zyk hebrajski niż t.zw. żydowski. », « Min. Grabski o szkolnictwie żydowskim (pisemna odpowiedź na pytania Naszego Przeglądu) », Nasz Przegląd, $1925, \mathrm{n}^{\circ} 141$, p. 3.

14. «Żargon », Ilustrowana Encyklopedja Trzaski, Everta i Michalskiego, opracowana pod redakcjĄ Dra Stanisława Lama, nakł. KsiĘgarni Trzaski, Everta i Michalskiego, Warszawa, cop. 1928, vol. 5 (Si-Z), col. 1215.

15. «Hebrajska literatura », ibid. vol. 2 (F-K), cop. 1928, col. 450.

16. Trzaski, Everta i Michalskiego Encyklopedja XX wieku, opracowana pod redakcją Dra Stanisława Lama, nakł. KsiĘgarni Trzaski, Everta i Michalskiego, Warszawa, [1937 ?], A-Z, 2119 p.

17. Ou encore «jĘzyk żydowsko-niemiecki » («langue judéo-allemande»), comme c'est le cas dans l'encyclopédie d'Orgelbrand dans l'article consacré à la littérature juive, cf. «Żydowska literatura ", Encyklopedyja powszechna, op. cit., t. 28, p. 1112.

18. Voir par exemple l'article dans le journal assimilationniste Izraelita : «O żargonie niemieckim i sposobach jego wytĘpienia » [ "Sur le jargon allemand et les moyens de l'éradiquer »,], Izraelita, Warszawa, 1887, n 5 , p. 33.

19. «Nie będzie Niemiec pluł nam w twarz / Ni dzieci nam germanił», Maria Konopnicka, «Rota », Przodownica, Kraków, novembre 1908. Mis en musique par Feliks Nowowiejski en 1910, ce chant fut extrêmement populaire (au retour de l'indépendance, il a failli être choisi pour l'hymne national polonais). Le poème a été inspiré par les événements à Września sous domination prussienne en 1901, où des enfants polonais ayant protesté contre l'enseignement de la religion en allemand avaient été durement réprimés, ce qui a entraîné une vague de protestations du côté polonais.

20. «I cóż bym wyrazić mogła w tej pokaleczonej niemczyźnie, pisanej hebrajskimi literami, którĄ my językiem żydowskim zowiemy? Nigdy Abraham, Izaak i Jakub, nigdy prawodawca nasz Mojżesz jŁ̨zykiem tym nie mówili. Rodowitym każdego językiem jest jŁ̨zyk tej ziemi, na której sį̨ człowiek urodził: my sį̨ rodzili w Polszcze, polska wiĘc mowa jest ojczystą mowĄ naszĄ; tĄ 
piszmy, w tej rozmawiajmy ", Julian Ursyn Niemcewicz, Lejbe i Siora czyli listy dwóch kochanków, romans, Klasyka mniej znana, Kraków, Universitas, 2004, p. 10.

21. Klemens Junosza, Tanie wydawnictwo dzieł Klemensa Junoszy, vol. X, Donkiszot żydowski : szkic z literatury żargonowej żydowskiej, Warszawa, nakł. Rodziny, 1899, p. 236.

22. Voir par exemple le poème de Wiktor Gomulicki, «El mole rachmim » (Poezje, Warszawa, 1886) par ailleurs pas du tout antisémite, où l'auteur décrit la scène du mariage juif : «Ils ont baragouiné dans leur langue, mais aucun n'a trouvé, comme Beaumarchais, la cérémonie ridicule » («Szwargotali, lecz obrzęd nie zdał się żadnemu / Uroczystością śmiesznĄ, jako Beaumarchais'emu »).

23. «Ils [les Juifs] baragouinaient toujours en jargon allemand, et n'ont pas cessé d'être des 'ashkénazes'« («Żargonem niemieckim szwargotali bezustannie, 'aszkenazami’ być nie przestali »), Teodor Jeske-Choiński, Historja Żydow w Polsce, Warszawa, nakł. Kasy Przezorności i Pomocy Warszawskich Pomocników KsiĘgarskich, 1919, p. 188.

24. «D'ailleurs, s'ils doivent absolument babiller en yiddish, qu'ils gardent leur addiction insensée, mais qu'ils daignent se confiner avec elle dans les cercles familiaux étroits, et là, en cachette, dans la discrétion de leur foyer, qu'ils baragouinent à satiété » («ZresztĄ, jeśli już koniecznie muszą paplać po żydowsku, niechaj zachowują swój nałóg bezsensowny, ale niechaj raczą przenieść siĘ z nim do ciasnych, najbliższych sobie kółek, i tam w ukryciu, w zaciszu domowym, szwargoczĄ do syta ». A. J. Cohn, « Kwestja żargonowa », Izraelita, Warszawa, 1887, $\mathrm{n}^{\circ} 29$, pp. 229-230. Voir aussi les titres «pièces en baragouin» («sztuki w szwargocie») et «littérature en baragouin» (« literatura szwargotowa»), Izraelita, 1886, pp.60-61. P. [S. H. Peltyn], « Coś o żargonie żydowskim », Izraelita, 1885, nº 28, p. 221 : « Ils baragouineront peut-être éternellement dans leur langue étrangère » («B̨̨dĄ może wiecznie szwargotać swĄ obca mowĄ »).

25. En 1900, un des journalistes d'Izraelita prend ouvertement ses distances envers cette appellation dans un article très favorable consacré à l'hebdomadaire cracovien Der Yid: "Le jargon de ces écrivains n'est pas un baragouin [szwargot], au contraire - il fait tout à fait l'impression d'être une langue, mais pas la langue allemande » («Żargon tych pisarzy to nie szwargot jakiś, przeciwnie - sprawia zupełnie wrażenie jŁ̨zyka, ale nie niemieckiego »), Leon W-W, « Prassa żargonowa », Izraelita, 1900, n 10, p. 114.

26. «Szwargot », SłownikjĘzyka polskiego... op. cit., 3e partie, vol. V, R-T, p. 579.

27. «Żydy, Szwaby, przekupki szwargoczĄ.», "Szwargot», Słownik jĘzyka polskiego Jana Karłowicza, Adama Kryńskiego i Władysława Niedźwiedzkiego, zeszyt 31-35, ułożony przez Władysława Niedźwiedzkiego z udziałem Kazimierza Króla, Warszawa, nakł. Prenumeratorów i Kasy im. Mianowskiego w druk. Gazety Handlowej, 1912, vol. 6, p. 693.

28. "Jargon: langage déformé, enlaidi, baragouin» («Żargon: jĘzyk zepsuty, zeszpecony, szwargot »), Słownik języka polskiego Jana Karłowicza..., op. cit., vol. 6 , p. 691.

29. "Szwargot: mowa gardłowa, nieprzyjemna i niezrozumiała, szczególnie żargon żydowski », M. Arcta Słownik ilustrowany..., op. cit., t. 2, p. 889.

30. Ibid., p. 1208.

31. Voir Katrin Steffen, Jüdische Polonität? Studien zur polnischsprachigen jüdischen Presse in der Zweiten Polnischen Republik (1918-1936), Inaugural-Dissertation zur Erlangung des Doktorgrades am Fachbereich Geschichts- und Kulturwissenschaften der Freien Universität Berlin, Berlin, 2002. Eugenia Prokop-Janiec, Międzywojenna literatura polsko-żydowska jako zjawisko kulturowe $i$ artystyczne, Kraków, Universitas, 1992.

32. C'était notamment le cas en Galicie, où l'utilisation du yiddish - et de l'hébreu - était interdite dans la vie publique (annonces, réunions, etc.). Bien que le ministre des affaires intérieures, Stanisław Wojciechowski, eut aboli cette loi par décret, des cas de l'utilisation de l'ancienne législation par des fonctionnaires zélés ont été rapportés. Encore en 1925, Nasz Przegląd rapporte 
la réponse du ministre Ratajski à l'interpellation du député juif Hartglas, dans laquelle ce dernier proteste contre l'interdiction de conférences en yiddish à Krzemieniec. Ratajski défend cette interdiction à deux reprises. Il interpréte l'article 109 de la constitution sur le droit d'utiliser la langue maternelle, en soutenant qu'il ne concerne que l'utilisation dans la sphère privée, cf. Nasz Przegląd, 1925, n 130, p. 4 et $n^{\circ} 142$, p. 3. Ce n'est qu'en 1927 que le ministre de l'intérieur Raczkiewicz envoie une circulaire aux autorités régionales où il se prononce contre l'interdiction de l'utilisation du yidish et de l'hébreu au cours de réunions. Nasz Przegląd, 1927, n 47, p. 5.

33. Voir par exemple l'article sur un projet gouvernemental concernant l'égalité des droits des langues minoritaires des confins (ukrainien, biélorusse, lituanien) avec le polonais (sur ces territoires). Nasz Przegląd s'aligne sur la protestation contre l'exclusion du yiddish de cette liste, déposée par une délégation du Club juif (Koło żydowskie) au Parlement polonais. L'article s'intitule « Le yiddish en tant que langue de 3e catégorie ». "J̨̨zyk żydowski jest jĘzykiem III-ej kategorji. Projekt rzĄdowy odmawia mu praw, ktore przyznaje jદ̨zykom mniejszości kresowych. », Nasz Przegląd, 1924, n 173, p. 6 et « Przeciw traktowaniu jĘzyka żydowskiego jako jĘzyka trzeciej kategorji ", Nasz Przegląd, 1924, nº 179, p. 1.

34. Voir par exemple Nasz Przegląd, 1927, $n^{\circ} 179$, p. 2.

35. Nasz Przegląd, 7 février 1930, p 5.

36. Dans sa critique de l'Encyclopédie Ultima Thule : «Je ne parle pas du fait que ces deux écrivains [Sholem-Aleykhem et Mendele Moykher-Sforim] ont écrit non pas en jargon, mais dans la vive et créative langue yiddish». ( Pomijam już sam fakt, że obaj wspomniani pisarze pisali nie w żargonie, lecz w żywym, twórczym języku żydowskim »). Marek Turkow, « Jak wygląda pierwsza polska encyklopedia powojenna », Nasz Przegląd, Warszawa, 1925, nº 185, p.4. Dans un autre article, Turkov rappelle l'engagement antisémite d'un des rédacteurs de l'encyclopédie, Franciszek Stanisław Michalski, voir Marek Turkow, «Encyklopedje polskie i ich stosunek do Żydow », Nasz Przegląd, 1926, n 93, p.6. Dans sa critique de l'encyclopédie Ilustrowana Encyklopedja Powszechna: "L'œuvre de Salomon [sic] Abramovitsh - écrivain en jargon (pour ces Messieurs, la langue yiddish est encore jargon - a été expédiée en trois lignes » («W trzech wierszach omówiono twórczość Salomona [sic] Abramowicza - pisarz żargonowy (dla tych panow jŁ̨zyk żydowski jest jeszcze żargonem [...] »), Marek Turkow, « Encyklopedje polskie i ich stosunek do Żydow », Nasz Przegląd, Warszawa, 1926, nº 93, p. 6.

37. «[...] a wiĘc wszystko w porzĄdku. 'Polskie radjo' opowiadajĄce Polsce i światu bajeczki antysemickie, nic nie słyszało o jĘzyku żydowskim, o jÆ̨zyku Asza, Szolom-Alejchema, Pereca, Lejwika. Dla polskiego radja niema jŁ̨zyka bogatej kultury żydowskiej, rozgał̨̨zionego szkolnictwa, poważnej wiedzy i teatru. Prawie cały świat kulturalny i polityczny uznał ten jĘzyk i jego prawa - tylko na fali 'warszawskiej' jest to wciĄż jeszcze ‘żargon polsko-rosyjsko-hebrajski ». Pierrot [Jakub Appenszlak], Nasz Przegląd, Warszawa, 1926, nº 250, p. 4.

38. «Społeczeństwo polskie zadawala się ogólnikami, bardzo czĘsto z czasów zamierzchłych. Jeżeli idzie naprz. o mniejszość żydowskĄ, społeczeństwo polskie w sposób lekceważĄcy traktuje jŁ̨zyk żydowski, znany wśród Polaków pod pogardliwą nazwą ‘żargon’« . «Polak o dĄżeniach narodowo-kulturalnych mas żydowskich: wywiad Naszego PrzeglĄdu z Dyrektorem Instytutu badań spraw mniejszości narodowych w Polsce, red. T. Hołowka », Nasz Przegląd, Warszawa, 12 janvier 1927, p. 6.

39. Voir par exemple l'entrée sur l'écrivain Sholem Asch dans l'Encyclopédie Ultima Thule: «Asz, Szalom, [...] écrit en jargon, que l'on appelle aujourd'hui 'nowożydowski' («[...] pisze w żargonie, czyli t. zw. obecnie nowożydowskim »), t. 1, p. 444.

40. Voir par exemple Zusman Segalowicz, Noce krymskie: nowele, przeł. z Yiddish'u Rachela Auerbach, Warszawa, Wydawnictwo Ch. Brzoza, 1936.

41. Voir par exemple l'article de l'historien et traducteur de la littérature yiddish en polonais, Samuel Hirszhorn au sujet du dictionnaire biographique des écrivains yiddish de Zalmen Reyzen. 
Hirszhorn critique le choix de Reyzen de n'inclure que les écrivains yiddish («jidyszowi ») en non pas juifs en général. (S. Hirszhorn, « Droga do niesmiertelnosci », Nasz Przegląd, Warszawa, 19 déc. 1927, p. 3). Le souci de précision semble aussi animer Hugo Bergman quand il parle de nécessité de créer une chaire pour le yiddish : «języka żydowskiego (jidyszu)» (Hugo Bergman, «Walka o katedrĘ dla żydowskiego », Nasz Przegląd, 1 janvier 1928, p.12), ou le traducteur de l'article d'Izaak Grynbaum : «Z wielkim trudem i mozołem mogła egzystować gazeta jidyszowa, nie mówiĄc już o hebrajskiej » (Izaak Grynbaum, « Asymilacja i polonizacja », Nasz Przegląd, 21 mars 1927, p. 3).

42. «J̨̨zyk. J̨̨zyk narodowy a dialekt », Świat $i$ życie : zarys encyklopedyczny wspótczesnej wiedzy $i$ kultury, red. Naczelny : Dr. Zygmunt Lempicki, red. Anna Chorowiczowa, KsiĄżnica - Atlas, Lwów Warszawa, vol. 2, D-K, 1934, col. 1079/1080.

43. « Idysz », ibid., vol. 5, A-Z, 1939, col. 777.

\section{RÉSUMÉS}

The complex attitude of Yiddish and Polish speakers, Jewish and non-Jewish, towards Yiddish, is reflected in the way the language is named. These designations evolve along the years, often following international - and particularly German - terminology. This evolution is not confined to a merely linguistic question: it reveals deep identity splits and is strongly linked to ideological and political choices. This article provides a detailed analysis of this evolution in newspapers and literature as well as in dictionaries and encyclopaedias. It explains the variations of point of view subtly implied by each terminological choice.

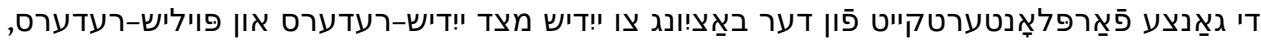

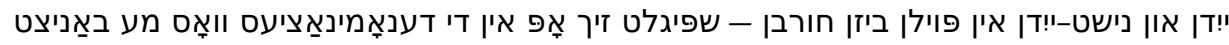

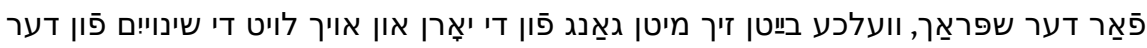

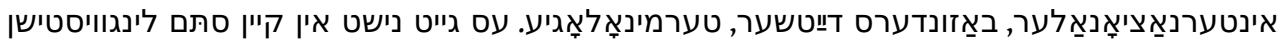

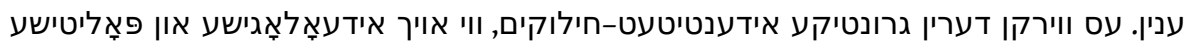

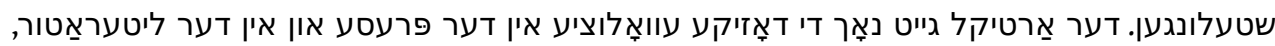

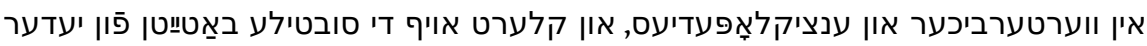

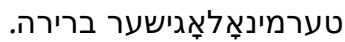


INDEX

Index chronologique : vingtième siècle

\section{מילות מפתח}

המאה העשרים, פולין, לשוני, ספרות, אידיש, מוכר בשפה, ז'רגון, יהודי פולין, ניב:

Mots-clés : yiddish (langue), langue familière, jargon, yiddishophone

Thèmes : littérature, linguistique

Index géographique : Pologne

Keywords : colloquial language, Polish Jews, jargon, dialect, Yiddish language, linguistics,

literature 